\title{
Sub-femtojoule optical modulation based on hybrid plasmonic devices
}

\author{
S. M. Sherif ${ }^{1}$ (D) M. A. Swillam ${ }^{1}$
}

Received: 23 June 2021 / Accepted: 24 December 2021 / Published online: 23 February 2022

(c) The Author(s) 2022

\begin{abstract}
Optical modulation is an essential process in the telecommunication technology. A subfemtojoule optical modulation based on low loss hybrid plasmonic waveguide which integrates silicon, metal, and electro-optic material is demonstrated. Modulation is achieved through applying modulating voltage across the electro-optic layers. Firstly, a directional coupler modulator is designed and simulated, it showed a modulation depth of $26 \mathrm{~dB}$, and energy consumption of $0.8 \mathrm{fJ} / \mathrm{bit}$. Secondly, a Mach Zender interferometer circuit based modulator is designed and analyzed, with a modulation depth of $32 \mathrm{~dB}$, and energy consumption of $0.8 \mathrm{fJ} / \mathrm{bit}$.
\end{abstract}

Keywords Optical modulators $\cdot$ Hybrid plasmonics $\cdot$ Directional couplers $\cdot$ Mach-Zender interferometers

\section{Introduction}

The accelerated developments in the telecommunication industry require optical modulators with enhanced performances. The desired properties of optical modulators are high speeds, large bandwidths, low losses and low power consumptions. Moreover, to be integrated with other photonic components in photonic integrated circuits, optical modulators designs should be compact with minimized footprints. In the attempt to develop fast responding Electro-optical (EO) modulators, different platforms were investigated such as the silicon ( $\mathrm{Si})$, Silicon organic hybrid ( $\mathrm{SOH})$, and hybrid plasmonic platforms.

Silicon is the most established platform in the photonics industry due to its high index contrast with silicon dioxide $\left(\mathrm{SiO}_{2}\right)$, its low losses, and CMOS compatibility (Bogaerts et al. 2005). But, the inversion symmetry of the Si crystal lattice diminishes the EO effects in Si (Reed and G.T., 2008). Thus, the SOH platform was introduced to benefit from the strong wave guiding properties of $\mathrm{Si}$ and the EO effects of the integrated organic materials or electro-optic polymers EOPs (Leuthold et al. 2013). SOH modulators based on different devices (Mohamed et al. 2018; Pan et al. 2018; Wolf et al. 2018) were demonstrated.

M. A. Swillam

m.swillam@aucegypt.edu

1 Department of Physics, School of Sciences and Engineering, The American University in Cairo, New Cairo 11835, Egypt 
Plasmonics based modulators can be highly compact due to beating the classical diffraction limit (Gramotnev and Bozhevolnyi 2010), moreover, plasmonics are associated with high electromagnetic fields enhancement and localization at metal/dielectric interfaces (Maier and Atwater 2005), but they usually suffer from high propagation losses because of the intrinsic high free electron density. However, it is also possible to integrate plasmonic materials with strong EO materials to build the Plasmonic organic hybrid (POH) platform (Robinson et al. 2018; Koos et al. 2015). POH modulators based on directional couplers (Zografopoulos et al. 2016a; Thomaschewski et al. 2020), ring resonators (Zografopoulos et al. 1039; Sherif et al. 2019), and MZIs (Haffner et al. 2015) have shown promising performances.

In this work, we demonstrate sub-femtojoule optical modulation based on the hybrid plasmonic platform where we utilize the waveguide design from our previous work on a low loss waveguide structure which is composed of Si-Ag-EOP layers, where an optical modulator based on a racetrack resonator was demonstrated (Sherif et al. 2019). We hereby implement a more recently used EOP; the DLD-164 chromophore which is a high linear $\chi^{(2)}$ material (Haffner et al. 2015; Elder et al. 2014) in our waveguide structure. Furthermore, we extend the impact of the low loss hybrid plasmonic structure on sub-femtojoule modulation through the directional coupler, and MZI mechanisms.

\section{Hybrid plasmonic waveguide structure}

The hybrid plasmonic waveguide is built of multi-layers as shown in Fig. 1, where on top of the $\mathrm{Si}$ substrate, the layers are arranged from bottom to top as; $\mathrm{SiO}_{2}(\mathrm{BOX})$ Si-EOP-Ag-EOP-Si. The optimization of the waveguide in order to minimize insertion losses was demonstrated in Sherif et al. (2019). Hereby, we use the same optimized waveguide parameters such that waveguide width is $400 \mathrm{~nm}$, layers thicknesses are $: t_{S i(\text { bottom })}=140 \mathrm{~nm}, t_{E O P(\text { bottom })}=t_{A g}=t_{E O P(\text { top })}=20 \mathrm{~nm}$, and $t_{S i(t o p)}=160 \mathrm{~nm}$.

The fabrication of this device is feasible through the following steps; $\mathrm{a} \mathrm{SiO}_{2}$ substrate is patterned using photolithography and wet etching, then a Si layer is deposited on top of the patterned $\mathrm{SiO}_{2}$ using chemical vapor deposition. The EOP is spin coated to cover the below Si layer. This is followed by Ag sputtering on top of the EOP layer to form a three-stack structure. Another Si wafer with a top layer of EOP through spin coating is pressed with the three-stack structure in a bonding process. The thick Si layer now on top can be thinned using reactive ion etching.

Fig. 1 Hybrid plasmonic waveguide structure

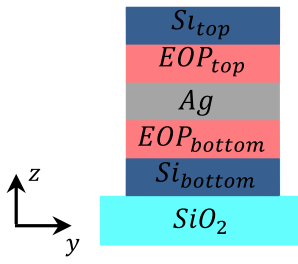


Fig. 2 Calculated hybrid plasmonic modes at $\lambda=1.55 \mu \mathrm{m}$; $\mathbf{a}$ symmetric mode, and $\mathbf{b}$ asymmetric mode

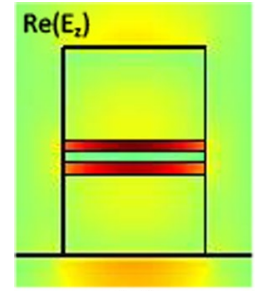

(a)

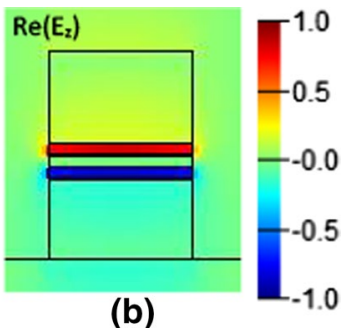

(b)

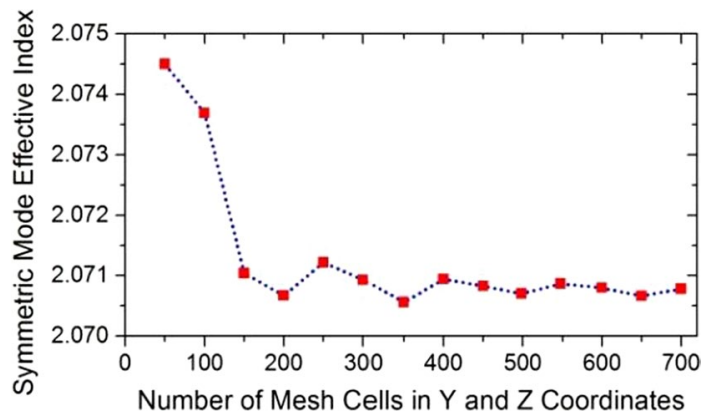

Fig. 3 Symmetric mode convergence with the number of mesh cells in $\mathrm{Y}$ and $\mathrm{Z}$ coordinates

\subsection{Waveguide modes}

When one the hybrid plasmonic waveguide is excited by a light source of wavelength in the near infra-red range with a central wavelength of $1.55 \mu \mathrm{m}$, the electromagnetic field interacts with the surface plasmons at the Ag/DLD-164 interfaces and the hybrid plasmonic mode propagates through the DLD-164 layers. A commercially available waveguide simulator was used for the calculation and characterization of the waveguide modes (https:// www.lumerical.com/products/mode/). Around the $1.55 \mu \mathrm{m}$ wavelength, the waveguide supports two hybrid plasmonic modes. Firstly, the symmetric mode is characterized by its electric field symmetric polarity on both sides of the Ag layer. Secondly, the asymmetric mode is characterized by its electric field opposite polarity on both sides of the $\mathrm{Ag}$ layer as observed from the electric field distributions of both modes in Fig. 2. Furthermore, the symmetric mode has a recognizable low loss of $0.015 \mathrm{~dB} / \mu \mathrm{m}$, and an effective index; $\mathrm{n}_{\text {eff }}=2.0707+0.0004 \mathrm{j}$, while the asymmetric mode has a higher loss of $0.75 \mathrm{~dB} / \mu \mathrm{m}$, and an effective index; $\mathrm{n}_{\mathrm{eff}}=3.005+0.02 \mathrm{j}$.

\subsection{Convergence testing}

Numerical calculations can show some fluctuations in the eigenmodes of the waveguides. These fluctuations may arise from different origins such as meshing of the structures, and effects of the simulation boundaries. Thus, it is desirable that the meshing resolution is high enough and the simulation boundaries are large enough in order to limit the numerical errors in the simulations (Chrostowski and Hochberg 2015). Figure 3 shows the convergence study of the symmetric mode with the $2 \mathrm{D}$ meshing, i.e., in both $\mathrm{Y}$ and $\mathrm{Z}$ coordinates. It can be observed that for low meshing accuracies the effective index values have higher 
Fig. 4 a SOI waveguide TM mode, b hybrid plasmonic waveguide symmetric mode

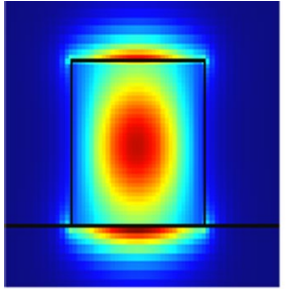

(a)

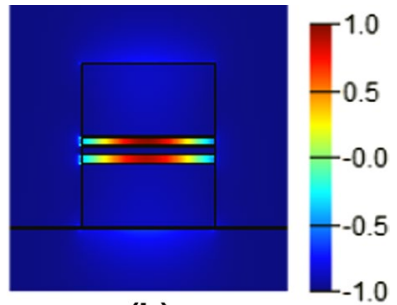

(b)

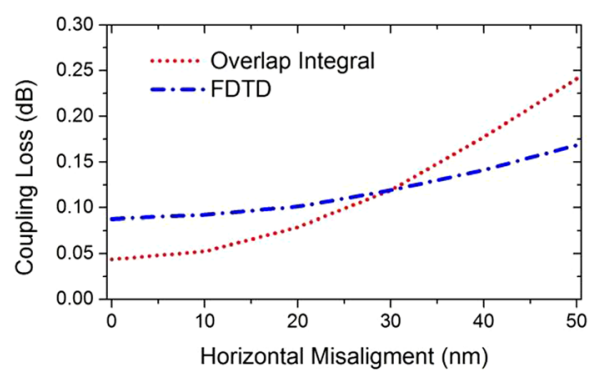

(a)

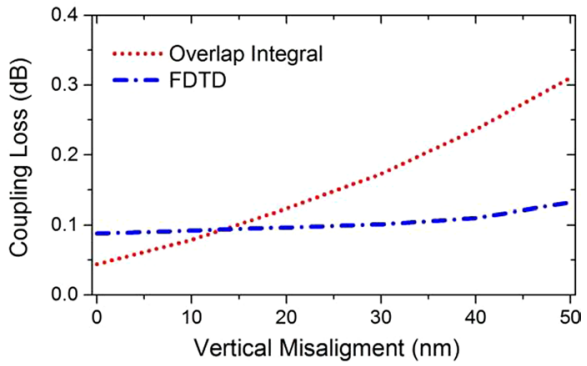

(b)

Fig. 5 Edge coupling misalignment loss calculated by the overlap integral and FDTD methods in the a horizontal, and $\mathbf{b}$ vertical directions

fluctuations, while for higher meshing accuracies (above 400 mesh cells) the effective index values converge to $\mathrm{n}_{\text {eff }}=2.0707$.

\subsection{Hybrid plasmonic mode coupling and misalignment loss}

The infrared TM mode of a conventional SOI waveguide of $400 \mathrm{~nm}$ width and $360 \mathrm{~nm}$ thickness is coupled to the hybrid plasmonic waveguide symmetric mode through edge coupling, the two modes are shown in Fig. 4.

The coupling loss due to misalignment between these two modes is calculated by the overlap integral based modal expansion techniques and FDTD methods as shown in Fig. 5. The two methods show close loss values with a small difference of only $0.1 \mathrm{~dB}$, and $0.2 \mathrm{~dB}$ for $50 \mathrm{~nm}$ misalignment in the horizontal and vertical directions, respectively. The deviation is due to the limited number of modes taken in the mode expansion techniques.

\section{Directional coupler modulator}

The directional coupler consists of two identical hybrid plasmonic waveguides separated by a distance s as shown in Fig. 6. The Ag layer serves as the electrode for applying the modulating voltage. The top and bottom Si layers are connected to $\mathrm{Ag}$ contacts, so to decrease the high resistivity of intrinsic $\mathrm{Si}$; we use a low n-doped $\mathrm{Si}$ of $10^{17} \mathrm{~cm}^{-3}$ carrier concentration, the Drude model parameters are based on (Gamal et al. 2015), with permittivity $\varepsilon_{S i}=11.7$, plasma frequency $\omega_{p}=3.56 \times 10^{17} \mathrm{rad} / \mathrm{s}$ and collision frequency 
Fig. 6 Directional coupler modulator design, a 3D view, and b cross-sectional view

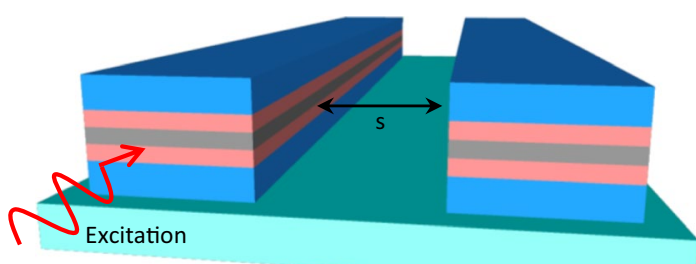

$\Gamma=9.7 \times 10^{8} \mathrm{rad} / \mathrm{s}$. The electro-optic polymer index variation with the applied voltage is given by Zografopoulos et al. 2016a; Sherif et al. 2019):

$$
\Delta n_{E O P}=0.5 n_{E O P}^{3} r_{33} \frac{V}{t_{E O P}}
$$

where $n_{E O P}=1.83$ is the DLD-164 index around the $1.55 \mu \mathrm{m}$ wavelength when no voltage is applied, $r_{33}=180 \mathrm{pm} / \mathrm{V}$ is the electro-optic coefficient of the DLD-164 chromophore (Zografopoulos et al. 1039; Haffner et al. 2015), $\mathrm{V}$ is the applied voltage, and $\mathrm{t}_{\mathrm{EOP}}$ is the thickness of the DLD-164 layer across which the voltage is applied.

When no voltage is applied across the DLD-164 layers, electromagnetic power is periodically coupled and exchanged between the two arms of the directional coupler, the coupling length is given by:

$$
l_{c}=\frac{\lambda}{2\left(n_{\text {even }}-n_{\text {odd }}\right)}
$$

where $\lambda$ is the operational wavelength, neven and nodd are the indices of the even and odd supermodes (Chrostowski and Hochberg 2015). When a small voltage is applied across the DLD-164 layers, its refractive index changes accordingly, thus, the supermodes indices also change, which results in a change in the coupling length given by (1).

This change in the coupling length affects the periodic exchange of power between the bar and cross arms, leading to a detectable optical power difference between the applied voltage levels. To maximize the difference in coupling length, the voltage is applied in a push-pull configuration such that the DLD-164 index increases in the bar, while it decreases in the cross. The transmission within the directional coupler is shown in Fig. 7 for both states when no voltage is applied and when a $1.5 \mathrm{~V}$ is applied in a push-pull configuration. A commercially available 3D FDTD Electromagnetic simulator was used for the

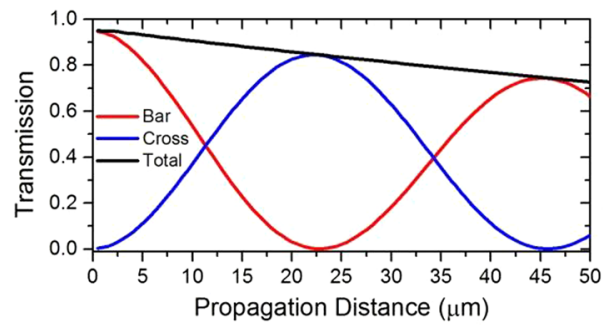

(a)

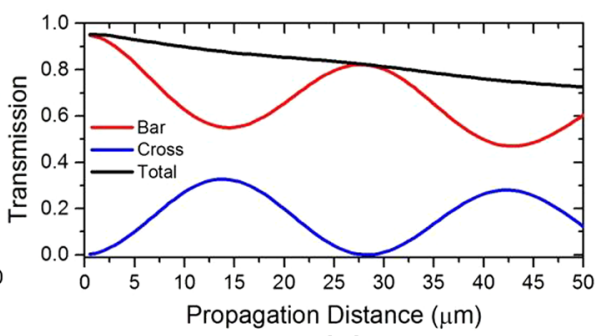

(b)

Fig. 7 Power exchange between directional coupler arms at $\lambda=1.55 \mu \mathrm{m}$, and at an aplied voltage of: a $0 \mathrm{~V}$, and $\mathbf{b} 1.5 \mathrm{~V}$ 
Fig. 8 Modulation depth for an applied voltage of $1.5 \mathrm{~V}$

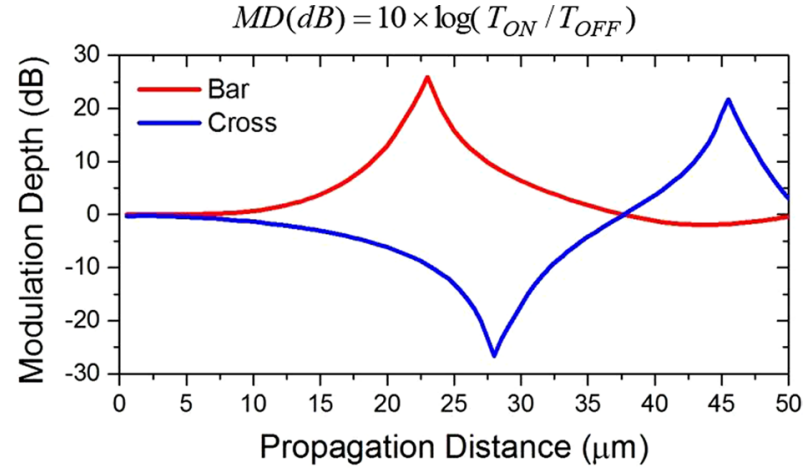

Fig. 9 Hybrid plasmonic mode profiles for: a $0 \mathrm{~V}, \mathbf{b} 1.5 \mathrm{~V}$ applied voltage

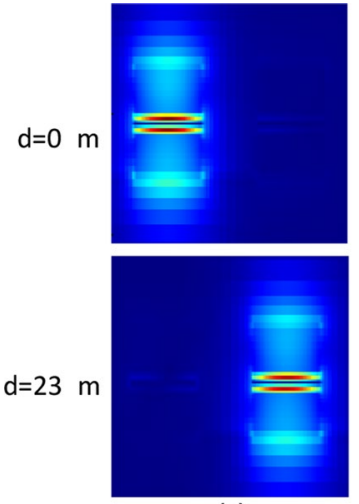

(a)

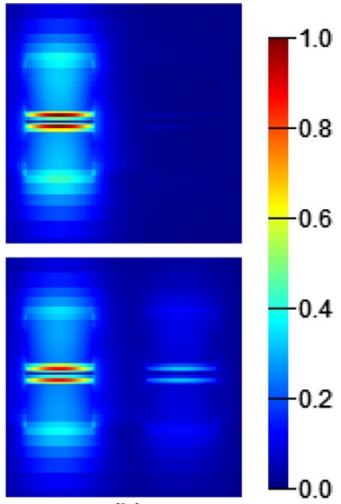

(b)

device design and simulation of light propagation in the directional coupler device (https:// www.lumerical.com/products/fdtd/).

\subsection{Modulation depth}

Modulation is realizable by measuring the optical power variation through both the bar and cross arms. Modulation depth is calculated using the transmission T for both the OFF and ON voltage states:

$$
M D(d B)=10 \times \log \left(T_{O N} / T_{O F F}\right)
$$

The modulation depth calculated for a propagation distance of $50 \mu \mathrm{m}$ is shown in Fig. 8, where it could be observed that for the cross arm, a modulation depth of $26 \mathrm{~dB}$ is achieved at a propagation distance of $23 \mu \mathrm{m}$ which corresponds to an insertion loss of $0.7 \mathrm{~dB}$, while a propagation distance of $26 \mu \mathrm{m}$ is needed to achieve a modulation depth of $26 \mathrm{~dB}$ through the bar arm with an insertion loss of $0.8 \mathrm{~dB}$, thus, for a more compact design, modulation through the bar arm would be favorable, such that the total footprint of the device is $23 \mu \mathrm{m}$ $\mathrm{X} 1.2 \mu \mathrm{m}$.

The hybrid plasmonic mode profiles at the excitation position $(\mathrm{d}=0 \mu \mathrm{m})$ and modulation position $(\mathrm{d}=23 \mu \mathrm{m})$ are shown in Fig. 9, where it can be observed that the in response to the applied voltage and associated change in coupling length, there has been a 
Fig. 10 Modulation depth for different applied voltages/DLD-164 index change

Fig. 11 Crosstalk between the directional coupler arms at $1.5 \mathrm{~V}$
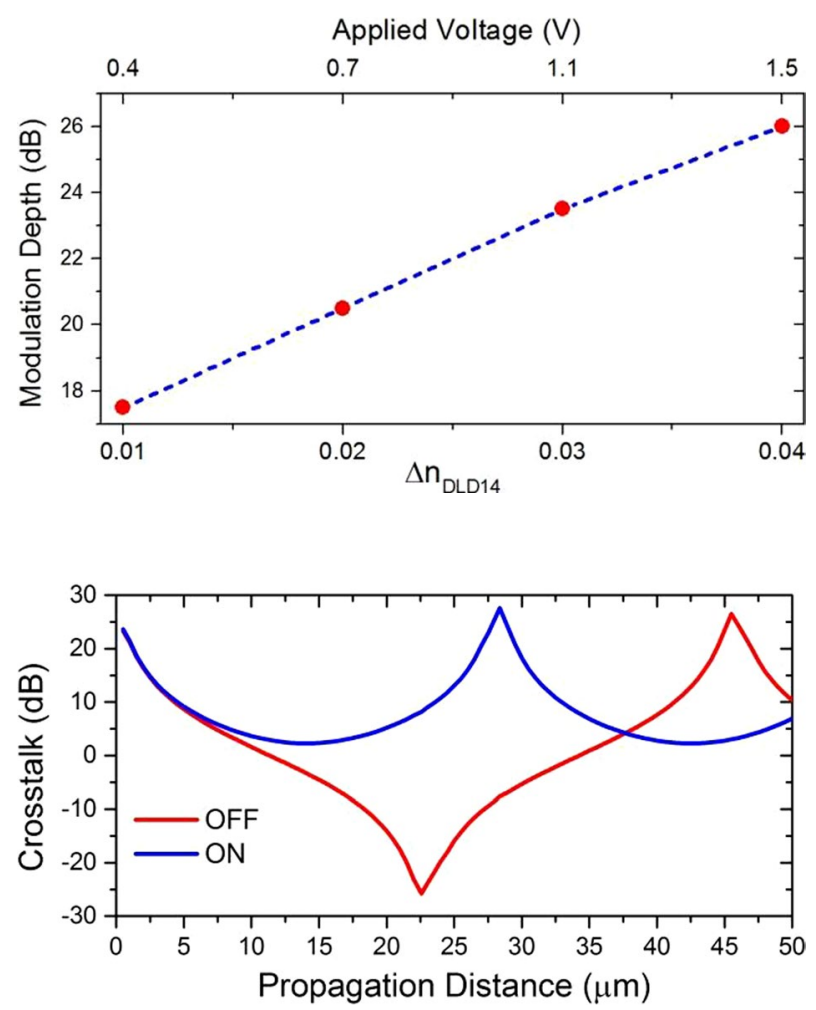

considerable change in the optical power in both the bar and cross arms. The modulation depth for different applied voltages/DLD-164 index change is shown in Fig. 10, where the increase in applied voltage results in an increased modulation depth.

\subsection{Cross talk}

The modulator crosstalk between the bar and cross arms is calculated for both OFF and ON states by:

$$
\text { crosstalk }=10 \times \log \left(T_{\text {cross }} / T_{\text {bar }}\right)
$$

It can be observed from Fig. 11 that the crosstalk is better than $25 \mathrm{~dB}$ for various operation positions, specially the $23 \mu \mathrm{m}$ propagation distance.

\subsection{Modulation speed}

The response time of the DLD-164, and the time constant of the RC circuit are the main factors that may limit the modulation speed. The response times of EOPs are in the range of femtoseconds (Haffner et al. 2015). To calculate the RC time constant, the capacitance C and the resistance $\mathrm{R}$ of the circuit needs to be calculated, the capacitance is given by: 


$$
C=\varepsilon_{o} \varepsilon_{D L D-164} \frac{A}{t_{D L D-164}}
$$

where $\mathrm{A}$ is the surface area of the DLD-164 layer which is equal to $0.4 \mu \mathrm{m} \mathrm{X} 15 \mu \mathrm{m}$ (modulation at a propagation distance of $15 \mu \mathrm{m}$ ). Thus, one DLD-164 layer has a capacitance of $9 \mathrm{fF}$, while the two parallel layers have a total capacitance of $18 \mathrm{fF}$. The resistivity of the $10^{17} \mathrm{~cm}^{-3} \mathrm{n}$-doped $\mathrm{Si}$ is equal to $0.087 \Omega \mathrm{cm}$ (Sherif et al. 2019). So, using:

$$
\begin{gathered}
R=\rho \frac{t_{D L D-164}}{A} \\
\text { speed }=\frac{1}{\tau}=\frac{1}{R C}
\end{gathered}
$$

The resistance of the $300 \mathrm{~nm}$ thick Si layers is $87 \mathrm{k} \Omega$, this results in a modulation speed that reaches $600 \mathrm{Gbit} / \mathrm{s}$. The power consumption is estimated by $P=2 C V^{2} f / 4$ (Zografopoulos et al. 2016b), thus, for an applied voltage of $0.3 \mathrm{~V}$ which results in $13 \mathrm{~dB}$ modulation depth (Fig. 9.), the energy consumption is limited to $0.8 \mathrm{fJ} / \mathrm{bit}$.

\section{Mach Zender modulator}

Optical modulators based on MZIs have shown high speed with low insertion losses (Haffner et al. 2015; Sato et al. 2017). In this section, we explore the potential of our low loss hybrid plasmonic waveguide to function as an optical modulator based on MZI. The hybrid plasmonic waveguide properties (effective index, loss) were exported from the waveguide simulator and mode solver (https://www.lumerical.com/products/mode/) and imported to a photonic integrated circuit simulator (https://www.lumerical.com/products/interconne ct/) to design the MZI optical modulator. The implemented S-parameters of the Y-couplers and the TM grating couplers were experimentally verified by https://github.com/SiEPIC/ SiEPIC_EBeam_PDK/wiki/Installation-instructions. Thus, a full imbalanced MZI circuit (Fig. 12) was built and simulated where the lengths of the two arms are $5 \mu \mathrm{m}$ and $15 \mu \mathrm{m}$. The change in the applied voltage results in a change in the effective index which shifts the interferences spectral positions as shown in Fig. 13. Modulation is thus realized due to the intensity change at the operational wavelength.

(a)

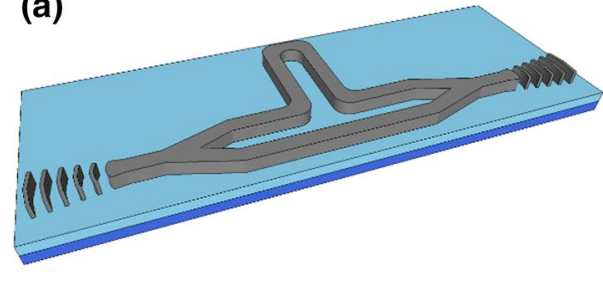

(b)

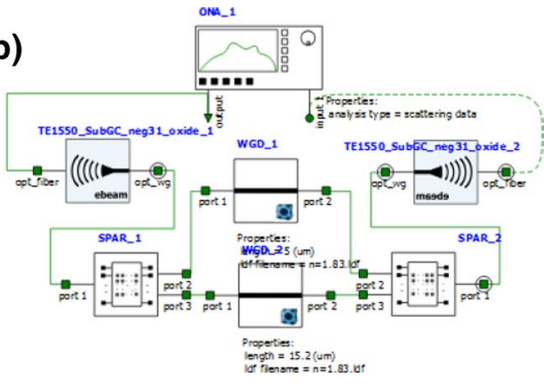

Fig. 12 Mach Zender Interferometer Circuit Modulator; a 3D view, b Simulated circuit 
Fig. 13 MZI transmission at different DLD-16 indices differences

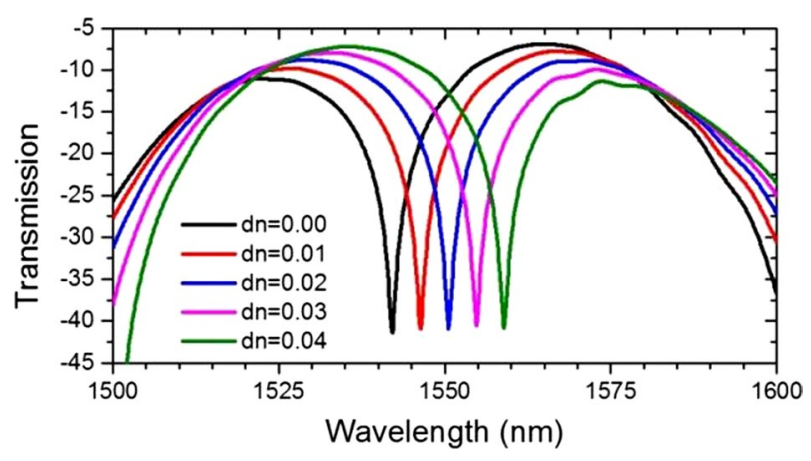

The modulation depth around the $1.55 \mu \mathrm{m}$ wavelength for an applied $1.5 \mathrm{~V}$ reaches $34 \mathrm{~dB}$, with a total insertion loss of $8 \mathrm{~dB}$. This insertion loss is larger than that of the discussed directional coupler due to implementing a full circuit with real experimental losses of the Y-couplers, and the grating couplers. The energy consumption is limited to $0.8 \mathrm{fJ} / \mathrm{bit}$ for a modulation depth of $24 \mathrm{~dB}$.

\section{Conclusions}

A hybrid plasmonic waveguide was used to build low power consumption optical modulators based on directional coupler and MZI mechanisms. The low loss of the excited symmetric hybrid plasmonic mode results in sub femtojoule power consumption per bit for the modulators operation with a modulation depth of $26 \mathrm{~dB}$.

Funding Open access funding provided by The Science, Technology.

\section{Declarations}

Conflict of interest The authors declare that there is no conflict of interests.

Open Access This article is licensed under a Creative Commons Attribution 4.0 International License, which permits use, sharing, adaptation, distribution and reproduction in any medium or format, as long as you give appropriate credit to the original author(s) and the source, provide a link to the Creative Commons licence, and indicate if changes were made. The images or other third party material in this article are included in the article's Creative Commons licence, unless indicated otherwise in a credit line to the material. If material is not included in the article's Creative Commons licence and your intended use is not permitted by statutory regulation or exceeds the permitted use, you will need to obtain permission directly from the copyright holder. To view a copy of this licence, visit http://creativecommons.org/licenses/by/4.0/.

\section{References}

Bogaerts, W., et al.: Nanophotonic waveguides in silicon-on-insulator fabricated with CMOS technology. J. Light. Technol. 23(1), 401-412 (2005)

Chrostowski, L., and Hochberg, M.: "Silicon photonics design: from devices to systems," Cambridge University Press, 2015. 
Elder, D.L., Benight, S.J., Song, J., Robinson, B.H., Dalton, L.R.: Matrix-assisted poling of monolithic bridge-disubstituted organic NLO chromophores. Chem. Mater. 26(2), 872-874 (2014)

Gamal, R., Ismail, Y., Swillam, M.A.: Silicon waveguides at the mid-infrared. J. Light. Technol. 33(15), 3207-3214 (2015)

Gramotnev, D.K., Bozhevolnyi, S.I.: Plasmonics beyond the diffraction limit. Nat. Photon. 4(2), 83-91 (2010)

Haffner, C., et al.: All-plasmonic Mach-Zehnder modulator enabling optical high-speed communication at the microscale. Nat. Photon. 9(8), 5-528 (2015)

https://github.com/SiEPIC/SiEPIC_EBeam_PDK/wiki/Installation-instructions

https://www.lumerical.com/products/fdtd/

https://www.lumerical.com/products/interconnect/

https://www.lumerical.com/products/mode/

Koos, C., et al.: Silicon-organic hybrid (SOH) and plasmonic-organic hybrid (POH) integration. J. Light. Technol. 34(2), 256-268 (2015)

Leuthold, J., et al.: Silicon-organic hybrid electro-optical devices. IEEE J. Sel. Top. Quantum Electron. 19(6), 114-126 (2013)

Maier, S.A., Atwater, H.A.: Plasmonics: Localization and guiding of electromagnetic energy in metal/dielectric structures. J. Appl. Phys. 98(1), 10 (2005)

Mohamed, S., Shahada, L., Swillam, M.: Vertical silicon nanowires based directional coupler optical router. IEEE Photonic. Tech. L. 30(9), 789-792 (2018)

Pan, Z., et al.: High-speed modulator based on electro-optic polymer infiltrated subwavelength grating waveguide ring resonator. Laser Photonics Rev. 12(6), 1700300 (2018)

Reed, G.T.: "Silicon photonics: the state of the art," John Wiley \& Sons, 2008.

Robinson, B.H., et al.: Optimization of plasmonic-organic hybrid electro-optics. J. Light. Technol. 36(21), 5036-5047 (2018)

Sato, H., Miura, H., Qiu, F., Spring, A.M., Kashino, T., Kikuchi, T., Ozawa, M., Nawata, H., Odoi, K., Yokoyama, S.: Low driving voltage Mach Zehnder interference modulator constructed from an electrooptic polymer on ultra thin silicon with a broadband operation. Opt. Express 25(2), 768-775 (2017)

Sherif, S.M., Elsayed, M., Shahada, L.A., Swillam, M.A.: Sub-Femtojoule hybrid plasmonic optical modulator. IEEE Photonics J. 11(4), 1-12 (2019)

Thomaschewski, M., Zenin, V.A., Wolff, C., Bozhevolnyi, S.I.: Plasmonic monolithic lithium niobate directional coupler switches. Nat. Commun. 11(1), 1-6 (2020)

Wolf, S., et al.: Silicon-organic hybrid (SOH) Mach-Zehnder modulators for $100 \mathrm{Gbit} / \mathrm{s}$ on-off keying. Sci. Rep. 8(1), 1-13 (2018)

Zografopoulos, D.C., Swillam, M.A., Shahada, L.A., Beccherelli, R.: Hybrid electro-optic plasmonic modulators based on directional coupler switches. Appl. Phys. A 122(4), 344 (2016a)

Zografopoulos, D.C., Swillam, M., Beccherelli, R.: Hybrid plasmonic modulators and filters based on electromagnetically induced transparency. IEEE Photonic. Tech. L. 28(7), 818-821 (2016b)

Zografopoulos, D.C., Swillam, M.A., Beccherelli, R.: Electro-optic modulators based on hybrid plasmonic micro-ring-disk resonators with femtojoule switching energy. Appl. Phys. A 122(12), 1039 (2016)

Publisher's Note Springer Nature remains neutral with regard to jurisdictional claims in published maps and institutional affiliations. 